\title{
UM OVERVIEW SOBRE A DISSEMINAÇÃO DAS TECNOLOGIAS E-HEALTH
}

LUIZ PHILIPI CALEGARI - luizpcalegari@gmail.com UNIVERSIDADE FEDERAL DE SANTA CATARINA - UFSC

DIEGO DE CASTRO FETTERMANN - dcfettermann@gmail.com UNIVERSIDADE FEDERAL DE SANTA CATARINA - UFSC

\section{RESUMO}

O contínuo desenvolvimento de tecnologias aplicadas à saúde, sobretudo as tecnologias $e$ health, proporcionou uma melhoria na qualidade de vida dos pacientes ao mesmo tempo em que aumentou a expectativa dos mesmos por melhores tratamentos no sistema de saúde. Dessa forma, torna-se necessário a identificação de aplicações tecnológicas para os serviços de saúde. Para o presente estudo, foi realizada uma revisão da literatura com o objetivo de reunir estudos que apresentam aplicações das tecnologias e-health. Como resultado, a presente revisão apresenta a identificação de aplicações tecnológicas disseminadas na literatura de $e$-health.

Palavras chave: e-health; monitoramento remoto de saúde; tecnologias da informação; tecnologias da comunicação; healthcare 


\section{INTRODUÇÃO}

O interesse das pessoas em relação às questões de saúde tem estimulado o desenvolvimento de produtos tecnológicos direcionados ao healthcare (CHAU et al., 2019). Em geral, essas tecnologias apresentam entre seus objetivos proporcionar a acessibilidade universal da sociedade aos serviços de saúde (HIXSON; BRAVERMAN, 2020). Parte deste objetivo é atingido com a incorporação da internet aos sistemas de healthcare, por meio do gerenciamento da informação e comunicação buscam a melhoria da prestação de cuidados centrados no paciente (PENEDO et al., 2020).

O contínuo desenvolvimento de tecnologias aplicadas à saúde proporcionou uma melhoria na qualidade de vida dos pacientes ao mesmo tempo em que aumentou a expectativa dos mesmos por melhores tratamentos no sistema de saúde (GELLER et al., 2008; KALEEBI, 2017; PISON et al., 2006; TANTUCCI et al., 2018). A literatura reporta a utilização de aplicativos digitais associados à internet indicados para o tratamento e monitoramento de diversas limitações na saúde dos pacientes, tais como no sistema locomotor, doenças cardiovasculares, doenças metabólicas, síndromes de dores crônicas, doenças psiquiátricas, e doenças oftalmológicas (SAFI; DANZER; SCHMAILZL, 2019).

Como forma de melhorar o atendimento à saúde, novas tecnologias passaram a ser incorporadas aos sistemas de healthcare, a exemplo das tecnologias $e$-health. Para o presente estudo, foi realizada uma revisão da literatura com o objetivo de reunir os diferentes estudos sobre tecnologias $e$-health. Os resultados do presente estudo apresentam um panorama abrangente sobre a disseminação das aplicações de tecnologias $e$-health na literatura.

\section{TECNOLOGIAS E-HEALTH}

A partir do desenvolvimento de tecnologias de informação modernas o conceito de $e$-health passa a ser utilizado para identificar um ambiente de transação complexo para relações eletrônicas associadas à saúde (DUPLAGA; LESZCZUK; ZIELINSKI, 2006). O termo $e$ health mais amplamente difundido na literatura se refere à intersecção entre os campos de saúde pública, informática médica e negócios, que promovem o aprimoramento dos serviços de saúde por meio do avanço tecnológico (EYSENBACH, 2001). Esta visão mais ampla associada ao conceito de $e$-health está fundamentada em cinco elementos: disponibilização de conteúdo, garantia de conectividade, inclusão de mecanismos econômicos/comerciais, criação de redes de compartilhamento e suporte ao atendimento clínico (DUPLAGA; LESZCZUK; ZIELINSKI, 2006). As tecnologias e-health podem ainda estar subdivididas em domínios, tais quais 
Telessaúde (e Telemedicina), sistemas de informações clínicas, redes de informações regionais e nacionais integradas, sistemas não clínicos utilizados para saúde pública e educação de saúde, saúde móvel (m-health), saúde personalizada (p-health e automonitoramento) e integração e análise em grande escala de dados heterogêneos (big data) (PIOTROWICZ, 2017). A partir da necessidade de modernização dos elementos base e dos subdomínios relacionados às tecnologias e-health, é apresentado um novo paradigma denominado healthcare 4.0, ou smarthealth. Essa nova abordagem de e-health também pode ser caracterizado pela incorporação de três novas tecnologias aos sistemas de saúde: Internet of Things - apresenta um direcionamento na identificação digital e na comunicação entre máquinas, promovendo uma ampla e avançada conectividade entre os diversos dispositivos utilizados nos sistemas de saúde (ATZORI; IERA; MORABITO, 2010); Cloud Computing - tem por objetivo viabilizar o armazenamento e o processamento de informações sem a utilização de recursos presentes no dispositivo (MASIP-BRUIN et al., 2016); e Big Data - apresenta-se como uma importante ferramenta para extração das informações contidas nesses grandes volumes de dados complexos (ACETO; PERSICO; PESCAPÉ, 2020).

Baseado nos conceitos tecnológicos supra descritos, a FIGURA 1 apresenta uma proposta de organização estrutural das tecnologias de informação e comunicação aplicadas nos serviços de saúde. Esta apresentação se faz essencial a conceitualização precisa dos termos relacionados às tecnologias de informação presentes na literatura associados ao tema de healthcare. A partir da Figura 1 é possível observar que as tecnologias healthcare 4.0 são consideradas como base para os serviços de saúde remotos. A junção das tecnologias IoT com as tecnologias de computação em nuvem permitem uma maior conectividade dos dispositivos que possibilitam o conceito de computação onipresente (U-health). A ampla utilização desses dispositivos para serviços de Telessaúde e Automonitoramento fomenta o processo de geração de dados complexos. O Big Data Analytics, por sua vez, transforma a carga de dados complexos em informações e abastece os sistemas $e$-health com a finalidade de orientar/reorientar tarefas computacionais e tomadas de decisão. 


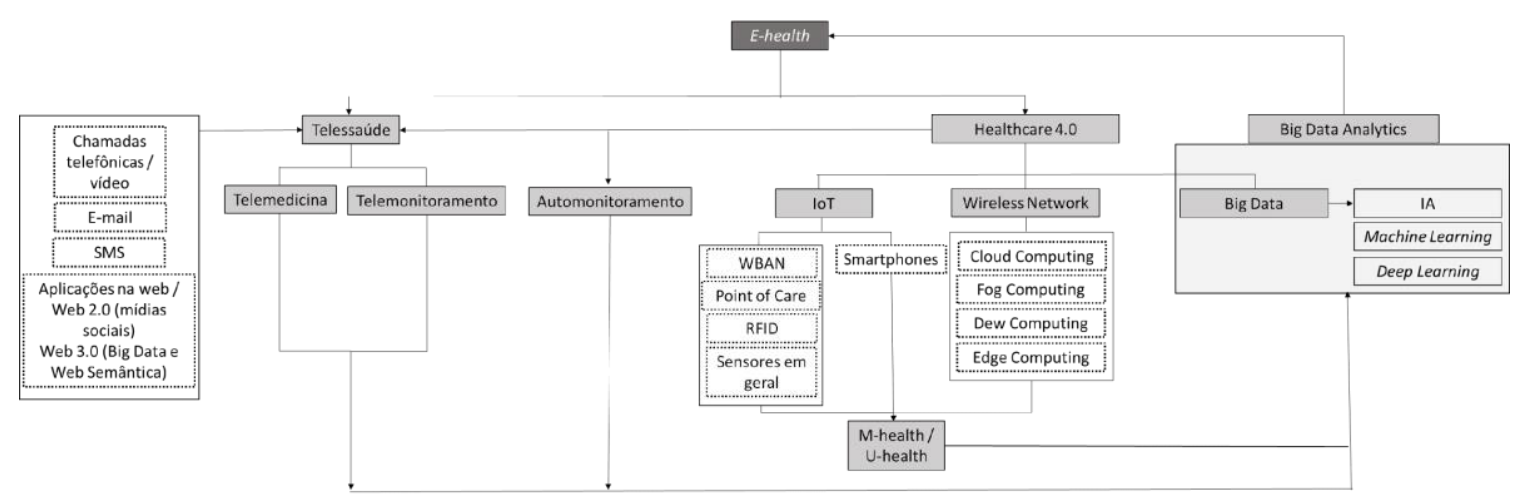

FIGURA 1 - Estrutura organizacional das tecnologias $e$-health

Fonte: Os autores

\section{METODOLOGIA}

Com o objetivo de identificar as oportunidades de pesquisa acerca do tema de e-health, foi adotado para a presente pesquisa o método de revisão sistemática da literatura, recomendada para reunião e análise dos estudos relevantes sobre o tema proposto (KITCHENHAM, 2004). A prática dessa metodologia permite a redução dos efeitos casuais, a limitação do erro sistemático e a confiabilidade para a análise de dados (REIM; PARIDA; ÖRTQVIST, 2015). Para elaboração da revisão sistemática, foi adotado a abordagem metodológica Preferred Reporting Items for Systematic Reviews and Meta-Analysis (PRISMA), utilizada originalmente para a área de medicina e expandido para outras áreas como ciências sociais e engenharia (AYODELE; CHANG-RICHARDS; GONZÁLEZ, 2020; MORENO-BLANCO et al., 2019). Por meio dos procedimentos recomendados pelo método PRISMA, e aplicados em estudos que relacionam saúde e tecnologia (BUDRIONIS; BELLIKA, 2016; DROSATOS; KALDOUDI, 2019), consideraram-se cinco etapas para a sistematização da revisão, conforme detalhado na FGURA 2:

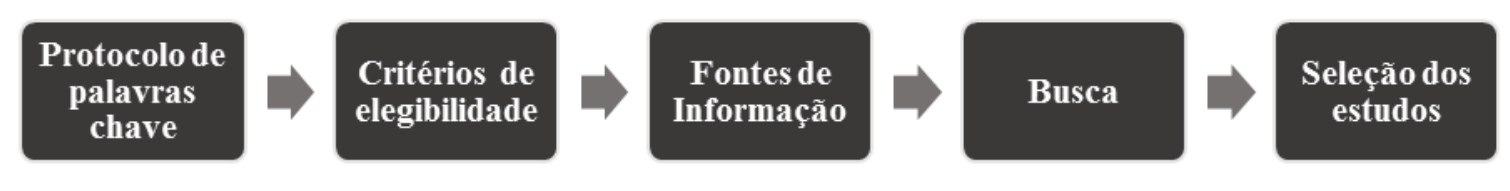

FIGURA 2 - Etapas para aplicação do PRISMA

Fonte: Os autores

As palavras-chaves, ou termos de pesquisa, possuem importância relevante para a representação de informações sobre o tema a ser explorado (BORBA; LAAN; CHINI, 2018). A escolha dos 
termos de pesquisa utilizados no procedimento de busca é essencial para identificar a literatura relevante de uma forma sistemática (STANSFIELD; O’MARA-EVES; THOMAS, 2017). Para tanto, foram incluídos três campos de pesquisa: o primeiro campo corresponde às aplicações tecnológicas na área de saúde, sobretudo aplicações de tecnologias de informação e comunicação em um contexto de $e$-health; o segundo campo de pesquisa refere-se às formas de monitoramento de saúde; O terceiro campo refere-se a aceitabilidade dos consumidores. A QUADRO 1 apresenta a estruturação da combinação das palavras-chave utilizadas na presente revisão. A busca foi realizada no período compreendido entre os meses de dezembro de 2020 e janeiro de 2021. Foram restringidos os campos de busca "título", "resumo" e "palavras-chave" dos artigos.

QUADRO 1 - Palavras-chave utilizadas para revisão sistemática da literatura

Fonte: Os autores

\begin{tabular}{|c|c|c|c|c|}
\hline Campo 1: saúde e tecnologia & Operador & $\begin{array}{l}\text { Campo 2: formas de } \\
\text { monitoramento de } \\
\text { saúde }\end{array}$ & Operador & $\begin{array}{l}\text { Campo 3: aceitabilidade e } \\
\text { aderência dos usuários }\end{array}$ \\
\hline $\begin{array}{l}\text { "e-health" OR } \\
\text { "Telemedicine" OR } \\
\text { "m-health" OR } \\
\text { ("internet of things" AND } \\
\text { "health") OR } \\
\text { ("wearable" AND "health") } \\
\text { OR } \\
\text { ("cloud computing" AND } \\
\text { "health") OR } \\
\text { ("edge computing" AND } \\
\text { "health") OR } \\
\text { ("fog computing" AND } \\
\text { "health") OR } \\
\text { ("dew computing" AND } \\
\text { "health") }\end{array}$ & AND & $\begin{array}{l}\text { "remote monitoring" } \\
\text { OR } \\
\text { "self-management*" } \\
\text { OR } \\
\text { "patient centered*" } \\
\text { OR } \\
\text { "self-monitoring*" } \\
\text { OR "user-centered*" }\end{array}$ & AND & $\begin{array}{l}\text { "user acceptance" OR } \\
\text { "user adoption" OR } \\
\text { "user engagement" OR } \\
\text { "user adherence" OR } \\
\text { "patient acceptance" OR } \\
\text { "patient adoption" OR } \\
\text { "patient engagement" OR } \\
\text { "patient adherence" OR } \\
\text { "provider adherence" OR } \\
\text { "provider acceptance" OR } \\
\text { "provider adoption" OR } \\
\text { "provider engagement" OR }\end{array}$ \\
\hline
\end{tabular}

Para o presente estudo, foram selecionados somente estudos que apresentaram aplicações de tecnologias de informação e comunicação para o monitoramento de saúde, nas bases de dados "Scopus", "Web of Science", "Pub Med", "Science Direct", "Emerald Insight” e "IEEE”. Foram ainda considerados somente os estudos de revisão de literatura publicados em periódicos e redigidos no idioma inglês. A estratégia de selecionar apenas as revisões sintetiza de forma coesa a reunião das informações relevantes sobre o tema de $e$-health e apresenta-se amplamente adotada na literatura da área de saúde (DIVAN et al., 2021; YAZDIZADEH et al., 2021). O detalhamento dos filtros considerados para seletividade dos estudos é apresentado na FIGURA 3. 




FIGURA 3 - Fluxo de informações processadas pelo PRISMA

Fonte: Os autores

\section{RESULTADOS E DISCUSSÃO}

Conforme os dados coletados, a FIGURA 4 apresenta um gráfico da quantidade de artigos considerados para este estudo, publicados por ano. Verifica-se um crescente número de publicações sobre o tema de $e$-health, indicando a atualidade e uma tendência de crescimento nos estudos sobre o tema.

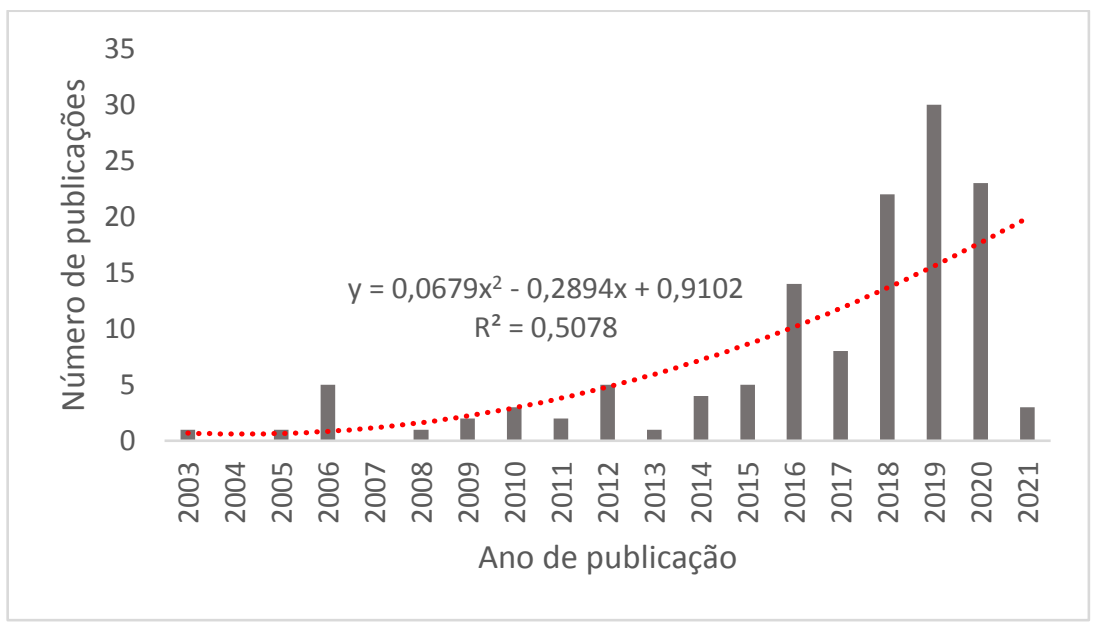

FIGURA 4 - Número de publicações por ano

Fonte: Os autores 
O Journal of Medical Internet Research, com 16 artigos, apresentou-se como o periódico com maior número de registros resultantes na presente revisão. Em segundo lugar, observa-se o periódico Telemedicine and e-health, com um total de 3 registros. Na sequência, oito outros periódicos apresentam 2 registros, enquanto todos os demais resultaram em apenas um registro acerca do tema.A análise dos periódicos confirma a dispersão dos estudos sobre $e$-health na literatura. São verificados periódicos de diversas áreas de concentração, com destaque para as áreas médicas e de ciência da computação. Quanto ao fator de impacto, verificamos que os periódicos com duas ou mais publicações resultantes na presente revisão apresentam média de seu fator de impacto $\left(\operatorname{SJR}_{\text {médio }}=1.111\right)$.

Os centros de estudo sobre o tema de $e$-health correspondem às instituições de filiação dos autores com publicações referenciadas nesta revisão de literatura. $\mathrm{O}$ número de publicações sobre o tema por país de filiação dos autores está representado na FIGURA 5. Verifica-se uma maior quantidade de publicações originadas dos Estados Unidos da América (62), seguido pelo Reino Unido (17) e Austrália (8), configurando-se nos países centro de estudos com maior quantidade de pesquisas sobre o tema.

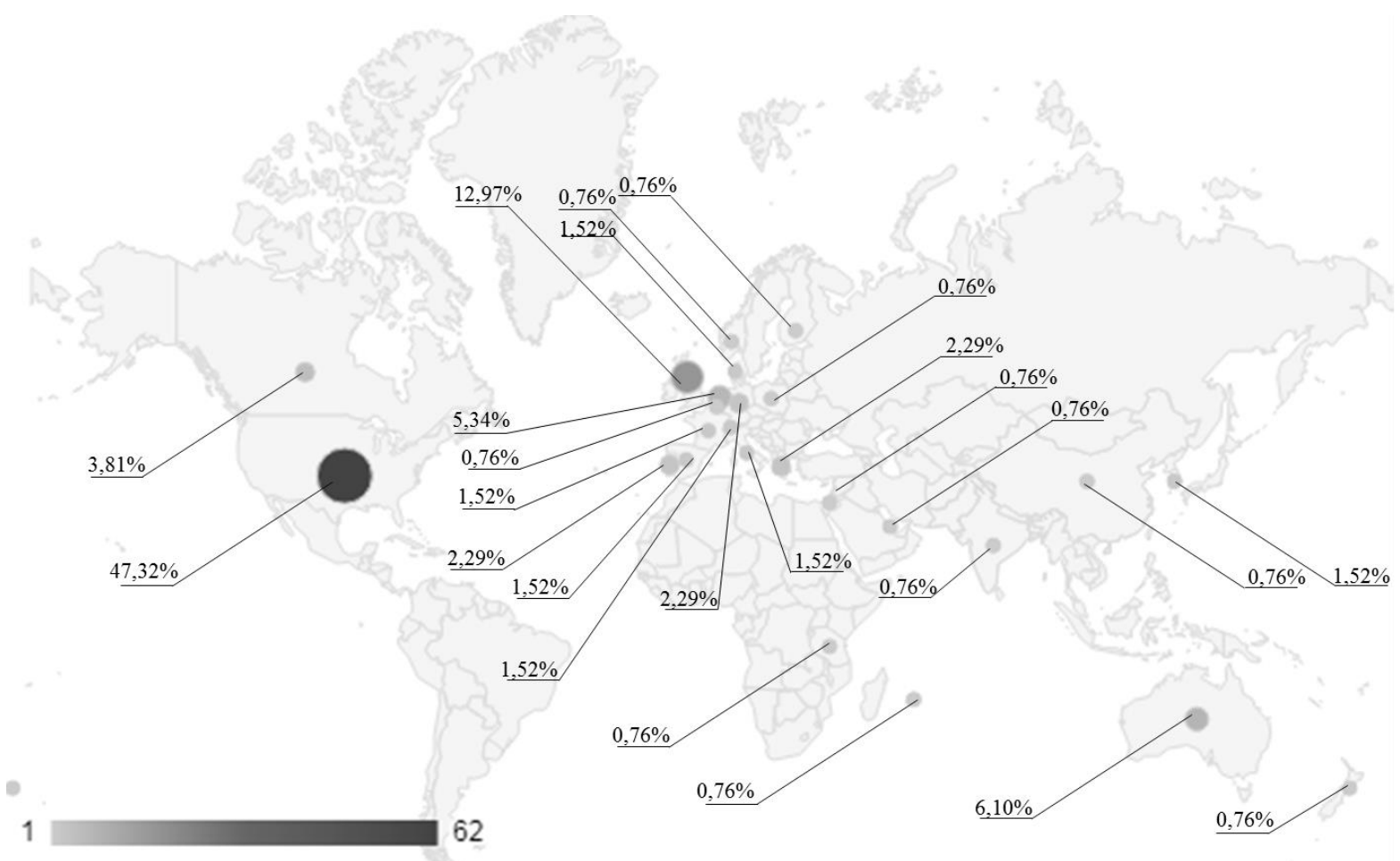

FIGURA 5 - Percentual do número de publicações sobre o tema por país

Fonte: Os autores 
Em termos absolutos, por meio do Quadro 2, é possível observar que a maioria dos estudos resultantes (62) empreendem em aplicações de e-health sem estabelecer foco em uma tecnologia específica. Em seguida, verifica-se a presença relevante de pesquisas direcionadas a aplicações de tecnologias associadas à m-health (24) e à Telemedicina (13). Entre os 30 artigos mais citados, também se verifica uma maior quantidade de estudos (11), com abordagens na aplicação de forma ampla das tecnologias $e$-health. Na sequência dos mais citados, apresentamse estudos focados em uma forma de tecnologia específica, tais quais Telemedicina (4), wearable (3), chamadas telefônicas (3) e m-health (3). O estudo de Delamater (2006), com o maior número de citações aborda a utilização de monitoramento remoto médico para o controle da diabetes por meio da utilização de chamadas telefônicas. O segundo estudo com maior número de citações (MICHIE et al., 2017) apresentou um overview sobre os desafios da utilização de sistemas $e$-health e fornece recomendações com a finalidade de orientar o progresso na pesquisa e prática de intervenções digitais na área da saúde.

Quadro 2 - Frequência das tecnologias $e$-health predominantes nos estudos

Fonte: Os autores

\begin{tabular}{|c|c|c|}
\hline \multicolumn{3}{|c|}{ Tecnologias predominantes nos estudos } \\
\hline E-health (62) & \multicolumn{2}{|l|}{ Geral (62) } \\
\hline \multirow{2}{*}{ Telessaúde (18) } & \multicolumn{2}{|l|}{ Geral (5) } \\
\hline & \multicolumn{2}{|l|}{ Telemedicina (13) } \\
\hline \multirow{7}{*}{ Healthcare 4.0 (42) } & \multicolumn{2}{|l|}{ Geral (1) } \\
\hline & \multirow{5}{*}{ IoT $(40)$} & Geral (6) \\
\hline & & M-health (24) \\
\hline & & Wearable (6) \\
\hline & & Smartphones (2) \\
\hline & & Outros sensores (2) \\
\hline & Big Data Analytics (1) & Machine learning (1) \\
\hline \multirow{4}{*}{ Tecnologias auxiliares (12) } & \multicolumn{2}{|c|}{ Chamadas telefônicas/ vídeo (6) } \\
\hline & \multicolumn{2}{|l|}{ Aplicações da web (3) } \\
\hline & \multicolumn{2}{|l|}{ e-mail/SMS (2) } \\
\hline & \multicolumn{2}{|l|}{ Blockchain (1) } \\
\hline
\end{tabular}

Apesar da quantidade relevante como foco na tecnologia $m$-health, constata-se que os estudos associados a essa tecnologia concentram-se no período de 2012 a 2021, enquanto os estudos 
correspondentes às classificações $e$-health e Telemedicina encontram-se dispersas ao longo de todo período analisado. Também é possível observar o declínio da quantidade de estudos sobre a realização de intervenções remotas por meio da utilização de chamadas telefônicas. Em contrapartida, também se torna importante destacar a introdução de estudos com a temática direcionadas ao healthcare 4.0, tecnologias de machine learning, blockchain e IoT somente a partir do ano de 2018. Dessa forma, por meio da FIGURA 6, é possível verificar evidências da atualização das tecnologias de informação e comunicação, a partir do desenvolvimento de novos dispositivos que possibilitam a evolução dos sistemas $e$-health.



FIGURA 6 - Timeline das Tecnologias presentes nos estudos resultantes da Revisão Sistemática

Fonte: Os autores

A partir dos resultados da análise bibliométrica, verifica-se um número crescente de publicações relacionadas ao tema de e-health. Além disso, diversos estudos apontam o crescimento da disseminação de tecnologias $e$-health entre os consumidores (CHAU, K Y et al., 2019; SHEGOG; BRAVERMAN; HIXSON, 2020). O crescimento do interesse de pesquisa, de desenvolvimento e de consumo dessas tecnologias indicam a atualidade sobre o tema $e$-health. Ao final da análise bibliométrica, ainda foi possível constatar a dispersão dos periódicos das publicações, sugerindo que o tema é estudado por diferentes áreas de conhecimento, tais quais desenvolvimento de tecnologias (ELLAHHAM, 2020; MOONIAN et al., 2020; WOLDAREGAY et al., 2019), alternativas de integração entre os sistemas de comunicação e os sistemas de saúde (ZHANG et al., 2020) e análise de aceitabilidade dos 
consumidores (ALZYOOD et al., 2018; BLACKWOOD et al., 2020). No entanto, verifica-se a carência de estudos que consolidem a eficácia de tratamentos realizados com essas tecnologias (MARKERT et al., 2021; ZHANG et al., 2020) e que compreendam a personalização dessas tecnologias com a finalidade de suprir necessidades específicas de diferentes consumidores (SHAN; SARKAR; MARTIN, 2019).

Também é importante destacar, a carência de publicações relevantes que considerem a percepção dos consumidores fora do eixo Europa-América do Norte, sobretudo em países com desenvolvimento tecnológico precário. Apesar da aceitabilidade dos consumidores do eixo Europa-América do Norte servir como ponto de referência, é necessário que a aceitabilidade dos consumidores seja analisada sob aspectos culturais, econômicos e políticos específicos de cada região.

\section{CONCLUSÃO}

Este estudo teve como objetivo identificar as tecnologias presentes na literatura acerca do tema de $e$-health, assim como as barreiras e benefícios para sua implementação. Foi realizada uma revisão sistemática da literatura, obtendo-se 130 registros considerados nesta pesquisa. Entre as tecnologias presentes na literatura, a maioria (62) não teve um foco em uma tecnologia em específico, abrangendo mais de uma tecnologia e-healht. Já de uma forma mais focada, destacam-se estudos direcionados nas tecnologias de m-health (24), telemedicina (13) e wearables (6). Outras tecnologias obtiveram resultados irrelevantes (machine learning, blockchain, IoT) ou que não apareceram nos estudos (Dew, Edge, Fog Computing). A provável insignificância resultante, deve-se ao fato de serem tecnologias recentes. No entanto, são tecnologias que possuem potencial de aplicação em sistemas e-health, as quais devem ser explorados em pesquisas futuras.

Apesar da diversidade de artigos correspondentes às tecnologias e-health, ainda é necessário compreender o efeito real dessas tecnologias perante o tratamento e a prevenção de doenças. Também se torna essencial a compreensão da percepção de consumidores com perfis diversificados perante a utilização dessas tecnologias e quais características dos dispositivos $e$ health apresentam-se imprescindíveis para o engajamento dos consumidores afim de monitorar sua saúde por meio da utilização dessas tecnologias.

\section{REFERÊNCIAS}

ACETO, G.; PERSICO, V.; PESCAPÉ, A. Industry 4.0 and health: internet of things, big data, 
and cloud computing for healthcare 4.0. Journal of Industrial Information Integration, v. 18, Feb. 2020. https://doi.org/10.1016/j.jii.2020.100129.

ALZYOOD, M.; JACKSON, D.; BROOKE, J.; AVEYARD, H. An integrative review exploring the perceptions of patients and healthcare professionals towards patient involvement in promoting hand hygiene compliance in the hospital setting. Journal of Clinical Nursing, v. 27, no. 7-8, p. 1329-1345, 2018. DOI 10.1111/jocn.14305.

ATZORI, L.; IERA, A.; MORABITO, G. The internet of things: a survey. Computer Networks, $\quad$ v. $54, \quad$ n. $15, \quad$ p. 2787-2805, Oct. 2010. https://doi.org/10.1016/j.comnet.2010.05.010.

AYODELE, O. A.; CHANG-RICHARDS, A.; GONZÁLEZ, V. Factors affecting workforce turnover in the construction sector: a systematic review. Journal of Construction Engineering and Management, v. 146, n. 2, Feb. 2020. DOI 10.1061/(asce)co.1943-7862.0001725.

BLACKWOOD, J; ARMSTRONG, M J; SCHAEFER, C; GRAHAM, I D; KNAAPEN, L; STRAUS, S E; URQUHART, R; GAGLIARDI, A R. How do guideline developers identify, incorporate and report patient preferences? An international cross-sectional survey. BMC Health Services Research, v. 20, n. 1, 2020. DOI 10.1186/s12913-020-05343-x.

BORBA, D. S.; LAAN, R. H.; CHINI, B. R. Keywords: convergences and differences with natural language and terminology. Perspectivas em Ciência da Informação, v. 24, n. 2, p. 3626, 19 Apr. 2018.

BUDRIONIS, A.; BELLIKA, J. G. The learning healthcare system: where are we now? A systematic review. Journal of Biomedical Informatics, v. 64, p. 87-92, 1 Dec. 2016. https://doi.org/10.1016/j.jbi.2016.09.018.

CHAU, K. Y.; LAM, M. H. S.; CHEUNG, M. L.; TSO, E. K. H; FLINT, S. W.; BROOM, D. R.; TSE, G.; LEE, K. Y.. Smart technology for healthcare: exploring the antecedents of adoption intention of healthcare wearable technology. Health Psychology Research, v. 7, n. 1, p. 80-99, Mar. 2019. https://doi.org/10.4081/hpr.2019.8099.

DIVAN, G.; BHAVNANI, S.; LEADBITTER, K.; ELLIS, C.; DASGUPTA, J.; ABUBAKAR, A.; ELSABBAGH, M.; HAMDANI, S. U.; SERVILI, C.; PATEL, V.; GREEN, J. Annual research review: achieving universal health coverage for young children with autism spectrum disorder in low- and middle-income countries: a review of reviews. Journal of Child Psychology and Psychiatry and Allied Disciplines, v. 62, n. 5, p. 514-535, May 2021. DOI: 
10.1111/jcpp.13404.

DROSATOS, G.; KALDOUDI, E. Blockchain applications in the biomedical domain: a scoping review. Computational and Structural Biotechnology Journal, v. 17, p. 229-240, 1 Jan. 2019. https://doi.org/10.1016/j.csbj.2019.01.010.

DUPLAGA, M.; LESZCZUK, M.; ZIELINSKI, K. Improving access of associated states to advanced concepts in medical telematics - a day before the accession to EU. 75., Mar. 2006. International Journal of Medical Informatics [...]. [S. l.: s. n.], Mar. 2006. v. 75, p. 300-305. https://doi.org/10.1016/j.ijmedinf.2005.08.014.

DELAMATER, A. M. Improving patient adherence. Clinical Diabetes, v. 24, n. 2, p. 71-77, 2006.

ELLAHHAM, S. Artificial Intelligence: the Future for diabetes care. American Journal of Medicine, v. 133, n. 8, p. 895-900, 2020. DOI 10.1016/j.amjmed.2020.03.033.

EYSENBACH, G. What is e-health? Journal of Medical Internet Research, v. 3, n. 2, p. 15, 18 Jun. 2001. DOI 10.2196/jmir.3.2.e20.

GELlER, G.; BERNHARDT, B. A.; CARRESE, J.; RUSHTON, C. H.; KOLODNER, K. What do clinicians derive from partnering with their patients? A reliable and valid measure of “personal meaning in patient care." Patient Education and Counseling, v. 72, n. 2, p. 293300, Aug. 2008. https://doi.org/10.1016/j.pec.2008.03.025.

GREIWE, J; NYENHUIS, S M. Wearable technology and how this can be implemented into clinical practice. Current Allergy and Asthma Reports, v. 20, n. 8, 2020. DOI 10.1007/s 11882-020-00927-3.

HIXSON, J D; BRAVERMAN, L. Digital tools for epilepsy: opportunities and barriers. Epilepsy Research, v. 162, 2020. DOI 10.1016/j.eplepsyres.2019.106233.

KALEEBI, J. N.. Risks related to poor infrastructure maintenance in urban local authorities in Uganda: a case study of Kamuli municipality. Kampala: Makerere University, 2017.

KITCHENHAM, Barbara. Procedures for performing systematic reviews. Keele: Keele University, 2004. (Keele University Technical Report TR/SE-0401).

MARKERT, C; SASANGOHAR, F; MORTAZAVI, B J; FIELDS, S. The use of telehealth technology to support health coaching for older adults: literature review. JMIR Human Factors, v. 8, n. 1, 2021. DOI 10.2196/23796. 
MASIP-BRUIN, X.; MARIN-TORDERA, E.; ALONSO, A.; GARCIA, J. Fog-to-cloud computing (F2C): the key technology enabler for dependable e-health services deployment. In: IFIP ANNUAL MEDITERRANEAN AD HOC NETWORKING WORKAHOP, 15., 2016, Vilanova ei la Geltru. Proceedins [...]. Piscataway: IEEE, 2016. DOI: https://doi.org/10.1109/MedHocNet.2016.7528425.

MICHIE, S; YARDLEY, L; WEST, R; PATRICK, K; GREAVES, F. Developing and evaluating digital interventions to promote behavior change in health and health care: recommendations resulting from an international workshop. Journal of Medical Internet Research, vol. 19, no. 6, 2017. DOI 10.2196/jmir.7126.

MOONIAN, O; JODHEEA-JUTTON, A; KHEDO, K K; BAICHOO, S; NAGOWAH, S D; NAGOWAH, L; MUNGLOO-DILMOHAMUD, Z; CHEERKOOT-JALIM, S. Recent advances in computational tools and resources for the self-management of type 2 diabetes. Informatics for Health and Social Care, v. 45, n. 1, p. 77-95, 2020. DOI 10.1080/17538157.2018.1559168.

MORENO-BLANCO, D; SOLANA-SÁNCHEZ, J; SÁNCHEZ-GONZÁLEZ, P; OROPESA, I; CÁCERES, C; CATTANEO, G; TORMOS-MUÑOZ, J M; BARTRÉS-FAZ, D; PASCUALLEONE, Á; GÓMEZ, E J. Technologies for monitoring lifestyle habits related to brain health: a systematic review. Sensors (Switzerland), v. 19, n. 19, 2019. DOI 10.3390/s19194183.

PENEDO, F J; OSWALD, L B; KRONENFELD, J P; GARCIA, S F; CELLA, D; YANEZ, B. The increasing value of eHealth in the delivery of patient-centred cancer care. The Lancet Oncology, vol. 21, no. 5, p. e240-e251, 2020. DOI 10.1016/S1470-2045(20)30021-8.

PIOTROWICZ, E. The management of patients with chronic heart failure: the growing role of e-Health. Expert Review of Medical Devices, v. 14, n. 4, p. 271-277, 2017. DOI 10.1080/17434440.2017.1314181.

PISON, Ulrich; WELTE, Tobias; GIERSIG, Michael; GRONEBERG, David A. Nanomedicine for respiratory diseases. European Journal of Pharmacology, v. 533, n. 1-3, p. 341-350, Mar. 2006. https://doi.org/10.1016/j.ejphar.2005.12.068.

REIM, W.; PARIDA, V.; ÖRTQVIST, D. Product-Service Systems (PSS) business models and tactics - a systematic literature review. Journal of Cleaner Production, v. 97, p. 61-75, 2015. SAFI, S; DANZER, G; SCHMAILZL, K J G. Empirical research on acceptance of digital technologies in medicine among patients and healthy users: questionnaire study. Journal of 
Medical Internet Research, v. 21, n. 11, 2019. DOI 10.2196/13472.

SHAN, R; SARKAR, S; MARTIN, S. S. Digital health technology and mobile devices for the management of diabetes mellitus: state of the art. Diabetologia, v. 62, n. 6, p. 877-887, 2019. DOI 10.1007/s00125-019-4864-7.

SHEGOG, R; BRAVERMAN, L; HIXSON, J D. Digital and technological opportunities in epilepsy: toward a digital ecosystem for enhanced epilepsy management. Epilepsy and Behavior, v. 102, 2020. DOI 10.1016/j.yebeh.2019.106663.

STANSFIELD, C.; O’MARA-EVES, A.; THOMAS, J. Text mining for search term development in systematic reviewing: a discussion of some methods and challenges. Research Synthesis Methods, v. 8, n. 3, p. 355-365, 1 Sep. 2017. DOI 10.1002/jrsm.1250. Available at: http://doi.wiley.com/10.1002/jrsm.1250.

TANTUCCI, A; RIPANI, C; GIANNINI, C; FREGONESE, M; DE ANGELIS, M. Can a program of food and diabetes education improve the quality of the lives of diabetic patients with a previous myocardial infarction? Ann Ig, v. 30, p. 120-127, 2018. https://doi.org/10.7416/ai.2018.2203.

WOLDAREGAY, A Z; ÅRSAND, E; WALDERHAUG, S; ALBERS, D; MAMYKINA, L; BOTSIS, T; HARTVIGSEN, G. Data-driven modeling and prediction of blood glucose dynamics: machine learning applications in type 1 diabetes. Artificial Intelligence in Medicine, v. 98, p. 109-134, 2019. DOI 10.1016/j.artmed.2019.07.007..

YAZDIZADEH, B.; WALKER, R.; SKOUTERIS, H.; OLANDER, E. K.; HILL, B. Interventions improving health professionals' practice for addressing patients' weight management behaviours: systematic review of reviews. Health Promotion International, v. 36, n. 1, p. 165-177, 1 Feb. 2021. DOI 10.1093/heapro/daaa039.

ZHANG, T; SODHRO, A H; LUO, Z; ZAHID, N; NAWAZ, M W; PIRBHULAL, S; MUZAMMAL, M. A joint deep learning and internet of medical things driven framework for elderly patients. IEEE Access, v. 8, p. 75822-75832, 2020. DOI 10.1109/ACCESS.2020.2989143.

ZHANG, Z.; BRAZIL, J.; OZKAYNAK, M.; DESANTO, K. Evaluative research of technologies for prehospital communication and coordination: a systematic review. Journal Of Medical Systems, v. 44, no. 5, 2020. https://doi.org/10.1007/s10916-020-01556-z. 
